\title{
Classification of Rigid and Deformable Objects Using a Novel Tactile Sensor
}

\author{
Alin Drimus, Gert Kootstra, Arne Bilberg and Danica Kragic
}

\begin{abstract}
In this paper, we present a novel tactile-array sensor for use in robotic grippers based on flexible piezoresistive rubber. We start by describing the physical principles of piezoresistive materials, and continue by outlining how to build a flexible tactile-sensor array using conductive thread electrodes. A real-time acquisition system scans the data from the array which is then further processed. We validate the properties of the sensor in an application that classifies a number of household objects while performing a palpation procedure with a robotic gripper. Based on the haptic feedback, we classify various rigid and deformable objects. We represent the array of tactile information as a time series of features and use this as the input for a k-nearest neighbors classifier. Dynamic time warping is used to calculate the distances between different time series. The results from our novel tactile sensor are compared to results obtained from an experimental setup using a Weiss Robotics tactile sensor with similar characteristics. We conclude by exemplifying how the results of the classification can be used in different robotic applications.
\end{abstract}

\section{INTRODUCTION}

For both humans and robots, tactile sensing is very important when manipulating tools or everyday objects, as well as for feature exploration and interaction. By extracting contact properties (position, forces, torques) in an exploration scenario, object properties such as geometry, deformability, and texture can be inferred. This can furthermore be used to improve grasping and manipulation of objects. Research in this area has gradually shifted from structured manufactured environments toward unstructured everyday environments. Visual feedback has proven to be an important source of sensory information necessary for grasp generation and control, [1], [2], [3], [4], [5], [6]. Although vision provides important information, it is not always trivial to obtain, and the accuracy is limited, due to imperfect calibration and occlusions. In addition, vision does not provide all information about object properties such as deformability or material properties. Errors in estimation of object shape are common even for known objects and these errors may cause failures in grasping. The different failures that may arise are difficult to prevent at the grasp-execution stage if the hand is only equipped with visual sensors. Tactile and finger-force sensors can be used as additional sensors to improve performance [7], [8], [9], but are still rather uncommon in practice. Mechanical compliance, for instance, is an important characteristic of an object, and it is essential in grasping fragile items. Humans

A. Drimus and A. Bilberg are with the Mads Clausen Institute for Product Innovation, University of Southern Denmark, 6400 Sønderborg, drimus@mci.sdu.dk, abi@mci.sdu.dk

G. Kootstra and D. Kragic are with the School of Computer Science and Communication Royal Institute of Technology(KTH), 10044 Stockholm, Sweden, kootstra@kth.se, danik@csc.kth.se furthermore use object properties such as hardness, thermal conductivity, friction and roughness in object manipulation, which could be addressed by robotic grippers as well by using haptic feedback.

In this paper, we propose the design of a novel tactile sensor that is flexible, has a sensitive output and is moreover cheap to manufacture. We demonstrate its use in a hapticbased object-classification scenario. The contributions of this paper are 1) describing the building of a tactile sensor prototype using piezoresistive materials and conductive thread electrodes, 2) a method for classifying rigid and deformable objects based on time series of features recorded from a palpation procedure. The system is implemented, evaluated, and finally compared to the widely used Weiss Robotics tactile sensor, [10].

This paper is organized as follows: Section II presents the related work regarding tactile sensors, while Section III describes the steps used to manufacture the proposed sensor prototype and the electronics used for data acquisition. Section IV describes the processing of the tactile images and the data modelling needed for object classification. The experiments are evaluated in Section V and the conclusions and further improvements are given in Section VI.

\section{RELATED WORK}

In terms of tactile-array sensors for static stimuli, such as pressure, there are a range of technologies that have been used with various results [11]. There are a few technologies that can be used for manufacturing tactile-array sensors, and the most used are piezoresistive (rubbers or inks), piezocapacitive, piezoelectrical, and optical [12]. [13] propose an industrial tactile-array sensor using a piezoresistive rubber. However, this sensor has a low spatial resolution and does not have any flexible capabilities. A flexible 16x16 sensor array with $1 \mathrm{~mm}$ spatial resolution was developed for minimal invasive surgery, but the sensor fails to give steady output for static stimuli, and has a high hysteresis and non-linearity [14]. A combination of static and dynamic sensors was developed in [15] to address both pressure profiles and slippage, but the design has only $4 \times 7$ cells, and a number of wires equal to the number of cells. Flexible sensors based on pressure conductive rubber with $3 \times 16$ cells were developed using a stitched electrode structure, but the construction method and the leak currents brought high variations in the measurements [16].

In terms of using tactile sensors to perform recognition or classification of objects, there are a few approaches. [17] used tactile information to estimate the state of an object in 
order to discriminate between different cans and filled bottles, and obtained similar results with the ones obtained from recognition tests done by humans. A different approach is described in [18], where multiple grasps are performed on a set of household objects. An unsupervised clustering method was used to learn a vocabulary from tactile observations and classification was done using a bag-of-words approach. The approach takes into considerations only tactile information at the points of contact with the considered objects. In some studies, grasp generation is based on visual input and tactile sensing is used for closed-loop control once in contact with the object. For example, the use of tactile sensors has been proposed to maximize the contact surface for removing a book from a bookshelf [19]. The application of force, visual, and tactile feedback to open a sliding door has been proposed in [20]. Tactile information can be also used to reconstruct the shape of unknown explored objects as proposed in [21].

One of the issues often faced in household scenarios are deformable objects. Planning grasps for these type of objects is not at all as well studied as rigid objects. Examples can be found in the literature, such as [22], where the deformation properties of objects are learned in order to apply suitable grasping forces for the associated objects.

Our work considers a tactile-array sensor based on piezoresistive technology. For classification, we look at the time series that the sensor provides during a full palpation procedure. Based on this dynamic information, different objects can be classified based on their tactile properties.

\section{THE TACTILE SENSOR}

\section{A. Building the tactile sensor}

After an early investigation and testing of different technologies and methods for building tactile sensors, presented in [23], we have chosen the piezoresistive principle (CSA) as the most suited to build a flexible tactile-array sensor. The CSA material has shown good performance in some research works related to finger-pads for robots [14]. This pressuresensitive conductive rubber is a material made of nonconductive elastomer, in which electric conductive particles are distributed/dispersed homogeneously. Since the electric conductive particles do not touch each other, electricity does not pass through the particles in the state when there is no external force. When external force acts, the particles come into contact with each other and more paths for a flowing current would be created. As a result of producing strain in the material with external force and of the percolation theory [14], the distribution state of the particles changes and the resistance of the material varies. The piezoresistive rubber shows an electrical resistance that ranges from about $0.5 k \Omega$ in the compressed (on) state to several $M \Omega$ in the free (off) state. Due to the percolation theory and internal distribution of particles, the Force-Resistance characteristic shows a non-linear behaviour and because of the elastomeric nature of the base material, it shows also hysteresis and creep effect. The characteristic of the material is depicted in Figure 1 for a number of 20 trials, where linearly increased force up to 400 grams-force and then decreased to 0 grams-force was applied to a test sample of the material. Taking into consideration the tip area of the actuator used to apply the force, the response for a cell ranges from approx. $30 \mathrm{kPa}(6$ psi) which is the threshold sensitivity up to $200 \mathrm{kPa}$ (30 PSI) being the upper limit before the creep effect appears. Even though non-linearities and creep effects are present in the behaviour of the material, we do not consider these as being a major disadvantage, as the human skin also shows them [14]. Given its flexibility, cost, sensitivity, robustness and ease of use we consider this material a very good candidate for building a tactile-array sensor.

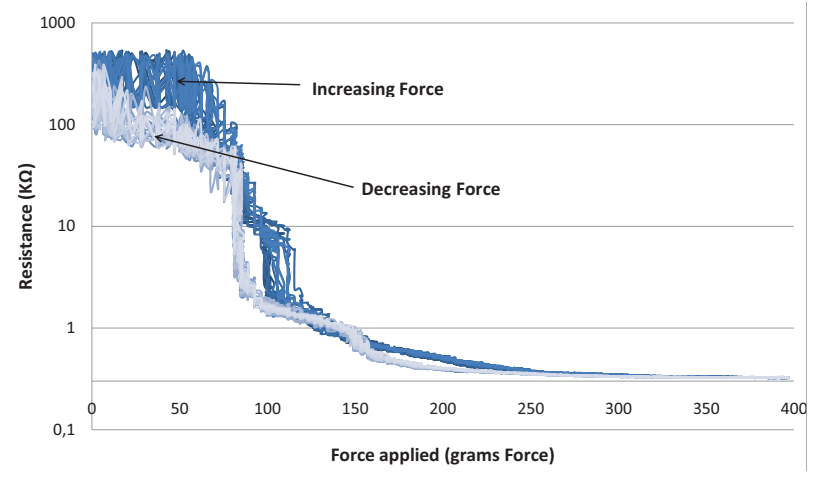

Fig. 1. Force vs. Resistance characteristic for the piezoresistive material, measured over 20 trials of applying increasing and decreasing force

In order to construct a tactile-array sensor, we seek inspiration in biology, especially in the characteristics of the human skin. Therefore, we are mainly interested in: dynamic range and sensitivity, size of taxel similar to the mechanoreceptor in the human hand, size of the array as big as possible without too much wiring complexity, robustness - to withstand repeated impacts and flexibility - so that we could apply this to any kind of robotic grippers, very much similar to an artificial skin. Other characteristics that we are aiming for are a good sensor output, low complexity and simple processing circuitry, ease of manufacture and a low price. Fulfilling these requirements is not easy, but our novel approach for making tactile sensors achieves most of our goals.

One of the biggest problems when processing the information from a multi-sensor device is the wiring complexity. When a high-resolution tactile array is the aim, with $n$ columns and $m$ rows - considering a pair of wires for each sensing elements, results in $n \times m \times 2$ wires. This means a complicated circuit design and other solutions may be better suited. One of them is by using electrodes arranged in rows on one side and as columns on the other side of the sensor device, as depicted in Figure 2. In this way, by selecting only one column and one row, the information from a single element can be read. Such a readout circuit would reduce the wiring from $n \times m \times 2$ to $n+m$ wires, which is a desirable improvement. This way it is possible to read a single sensor's value at a specific moment, resulting in high speeds for iteration through all columns and rows.

To build a tactile-array sensor, we start with a flexible 
substrate (PVC) covered by an adhesive layer. On top of this substrate, we lay conductive threads spaced $2.5 \mathrm{~mm}$ apart in a series of 8 parallel lines. On top of the thread, we add a piezoresistive rubber patch, which is $0.5 \mathrm{~mm}$ in thickness, and has a $20 \times 20 \mathrm{~mm}$ size. Next, a similar layer as the base layer is added on top, only this time, the conductive threads overlap the bottom ones perpendicularly. These steps of manufacturing are illustrated in Figure 3. The conductive threads ensure the flexibility and maximum compliance of the whole structure. From our previous results [23] we have concluded that there should not be any permanent electrical contact between the electrodes and the piezoresistive rubber patch, as this reduces the sensitivity for the low-forces range. The conductive threads show a resistance of about $10 \Omega$ per $10 \mathrm{~cm}$, which does not affect the performance of the sensor considering these threads have not more than $40 \mathrm{~cm}$ before the connector. The resulted prototype is roughly $25 \times 25 \mathrm{~mm}$, $1 \mathrm{~mm}$ in thickness and has a number of 64 taxels. We have manufactured larger arrays using this technique as well, but this is beyond the scope of this paper.

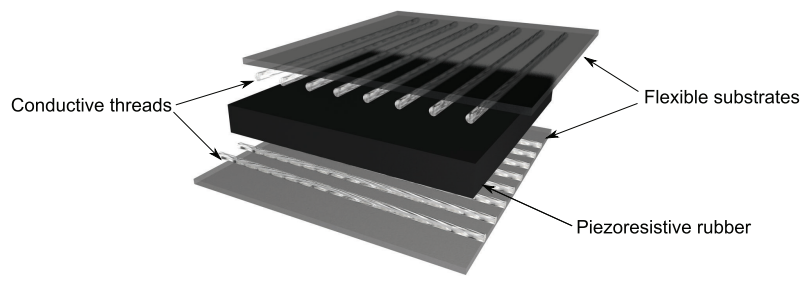

Fig. 2. Sensor structure
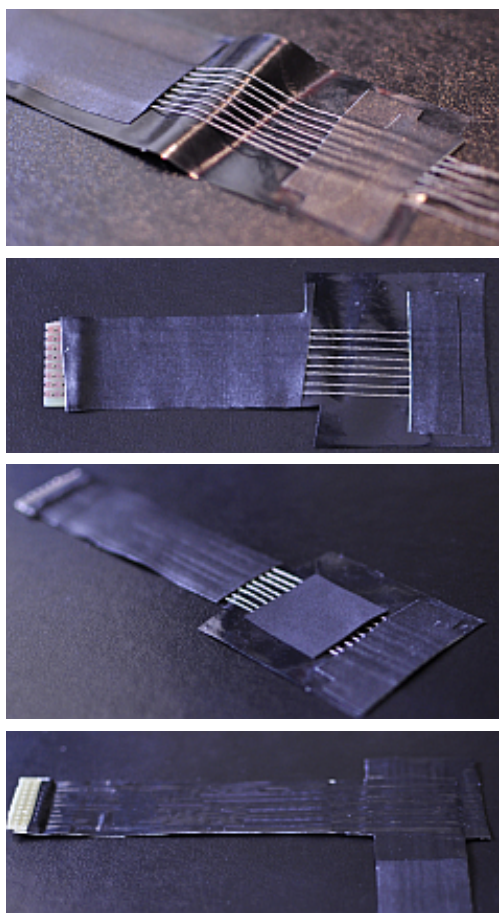

Fig. 3. Building the tactile sensor

\section{B. Data acquisition}

1) Signal conditioning: The signal conditioning for measuring the pressure applied over a tactile cell is based on the voltage divider principle. In the configuration described in Figure 4, the sensor cell is modelling a variable resistor with a value of up to $500 \mathrm{~K} \Omega$ and is connected in series with a fixed resistor - with a value typically less than $1 \mathrm{~K} \Omega$.

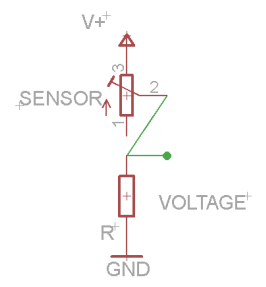

Fig. 4. Signal conditioning for measurement of one tactile cell

The voltage is measured over this resistance as: Voltage $=V_{+} \frac{R}{R_{\text {sensor }}+R}$ Based on this equation, a high pressure applied on the material will result in a low resistance value for the sensor, increasing the voltage drop over $R$. A low pressure applied on the material will result in a high resistance value for the sensor, therefore the voltage drop over $R$ approaching $0 \mathrm{~V}$.

2) Data acquisition: In order to achieve a high multiplexing speed and a high number of inputs we have used a dsPIC33FJ256, a 16 bit digital signal controller developed by Microchip.

Assuming an array of $n \times m$ elements arranged in $n$ rows and $m$ columns, the scanning procedure works as follows: for each $r_{0 w}$ from $\left(r o w_{1}, \ldots\right.$, row $\left._{n}\right)$ we apply a voltage over the $\operatorname{row}_{i}\left(V_{\text {applied }}\right)$ and all the others are kept to ground $(0 \mathrm{~V})$. We use $n$ Digital Output ports $\left(O_{1}, \ldots, O_{n}\right)$ to control which row will be enabled and which not, by setting $O_{i}=1$ and all others $O_{1}, \ldots, O_{i-1}, O_{i+1}, \ldots, O_{n}=0$. At this point, we use $m$ Analog Input ports $\left(I_{1}, \ldots, I_{m}\right)$ corresponding to the $m$ columns, column $n_{1}, \ldots$, column $_{m}$. We start by converting the voltage on $I_{1}$ by ADC, followed by the conversion of the $I_{2}$ and so forth, until we reach $I_{m}$. After this point we have obtained $m$ values, $V_{i 1}, V_{i 2}, \ldots, V_{i m}$ that represent the voltage difference between row $_{i}$ and all $m$ columns. Doing so will result in a matrix of $n \times m$ voltage readings. Each such matrix represents a frame or a tactile image.

The implemented data-acquisition module scans tactile arrays of up to 512 cells, providing 100 frames per second, with 8 bit data for each taxel. In our case, the module was used to scan the 64 taxels of the sensor and providing a tactile image every $10 \mathrm{~ms}$.

\section{OBJECT CLASSIFICATION}

The goal of the application is to classify the ten rigid and deformable objects shown in Figure 6 by performing a simple palpation procedure with a parallel gripper, using only the measured tactile feedback. The elastic properties (Young's modulus, stress-strain curve or Poisson Ratio) of the objects are not known a priori due to the fact that it is very difficult 


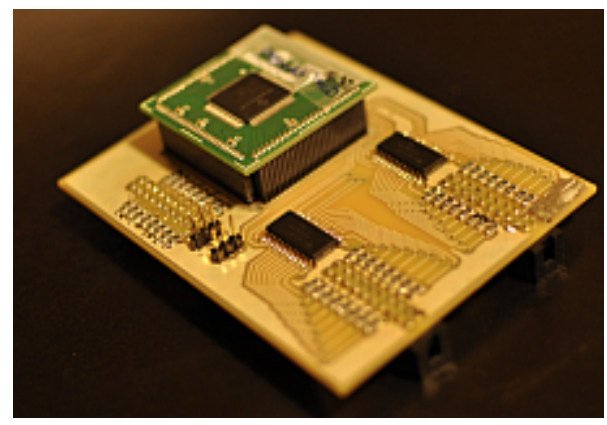

Fig. 5. Acquisition board

to determine such parameters given that the tested objects are not made of linear materials and have a non uniform structure and shape. The only constraints on the objects to be grasped is that they should be smaller in at least one dimension than the gripper opening. During the experiments, the objects were manually placed in between the gripper jaws. The palpation procedure started by closing the gripper's fingers until contact was established. This was then followed by a squeeze procedure, in five small steps, each step squeezing the object $1 \mathrm{~mm}$. Squeezing stiffer objects requires the use of more force, which translates to the increase of the current used by the gripper to perform the action. If the object cannot be further squeezed due to its stiffness, then the gripper would use its maximum rated force to squeeze it and this will be propagated onto the sensor, which will react accordingly, giving maximum output. After these squeeze steps, five desqueeze steps of $1 \mathrm{~mm}$ each were executed, and then the gripper released the object. The force applied by the gripper at its jaws is dependent on the material properties of the grasped object. The whole palpation procedure lasted about 6 to 7 seconds, depending on the object's size.

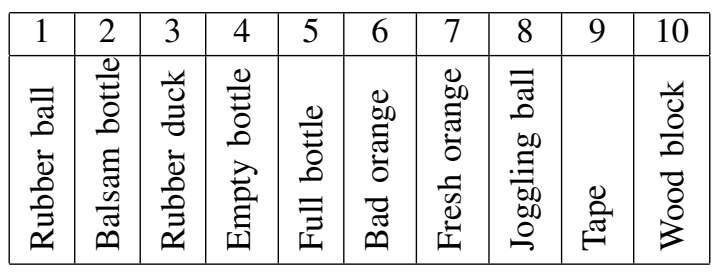

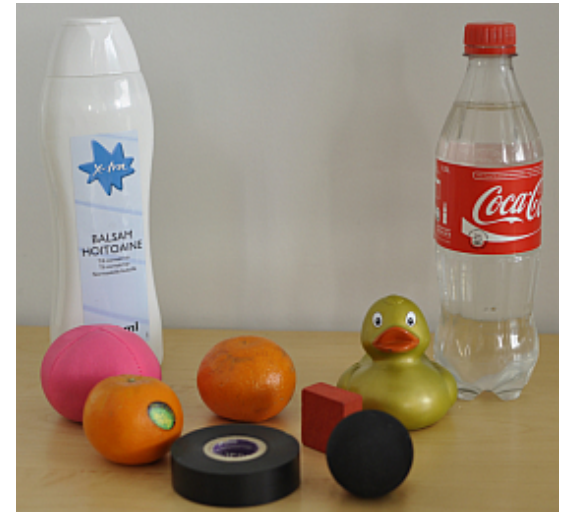

Fig. 6. Objects used in the experimental evaluation.

\section{A. Hardware Setup}

The hardware setup consisted of a Schunk PowerCube Robotic Arm with a 1-DOF Schunk PG70 parallel gripper as the end effector (see Figure 10). The parallel jaws were equipped with our tactile sensors, and then further connected to the data-acquisition circuitry as described in the previous section. The data-acquisition modules stream the data over USB to a host computer that records the tactile data and controls the execution of the grasp procedure.

\section{B. Data modelling}

The start of a palpation procedure (time $t_{0}$ ) is considered when both gripper jaws are in contact with the object, which is given when the tactile-sensor data is above a specified threshold. The procedure ends at time $t_{N}$, where $N$ represents the number of frames (tactile images) recorded from the tactile sensors. In our case the data-acquisition system provides a tactile image each $10 \mathrm{~ms}$. Examples of such tactile images are illustrated in Figure 8.a) - 8.e) for a few grasped objects, being sampled every $1 \mathrm{~s}$ from a palpation procedure. Because the execution time varies slightly for each experiment, the number of tactile images in each sequence varies between 500 and 520. A tactile image is an array of 64 values $(8 \times 8), x_{1}, x_{2}, \ldots, x_{64}$, each representing an 8 bit value that encodes the pressure applied over the taxel. Considering around 500 frames for each sensor during an exploration procedure, we obtain a high-dimensional description of each palpation procedure. In order to reduce the dimensionality, we extract just the first two moments of each tactile image as two independent features. The first feature corresponds to the average of an image, given by:

$$
\mu=\frac{1}{N} \sum_{i=1}^{N} x_{i},
$$

and the second feature corresponds to the standard deviation of the pixels in an image, given by:

$$
\sigma=\sqrt{\frac{1}{N} \sum_{i=1}^{N}\left(x_{i}-\mu\right)^{2}}
$$

The average of the tactile image gives a good estimate of the overall pressure applied to the contact area, which increases with the number of contact taxels and with the pressure over each taxel. The standard deviation is a rough estimate of the number of contact pixels, describing a wider or narrower contact area. These two features reduce the dimensionality of the data to a number of $N$ values for each feature. An observation $Z$ is therefore represented as:

$$
\begin{aligned}
& Z_{\mu}=\left\{\mu_{1}, \mu_{2}, \ldots, \mu_{N}\right\} \\
& Z_{\sigma}=\left\{\sigma_{1}, \sigma_{2}, \ldots, \sigma_{N}\right\}
\end{aligned}
$$

In Figure 7, different time series depicting the $\mu$ and $\sigma$ sequences for the Full Bottle object are represented. 


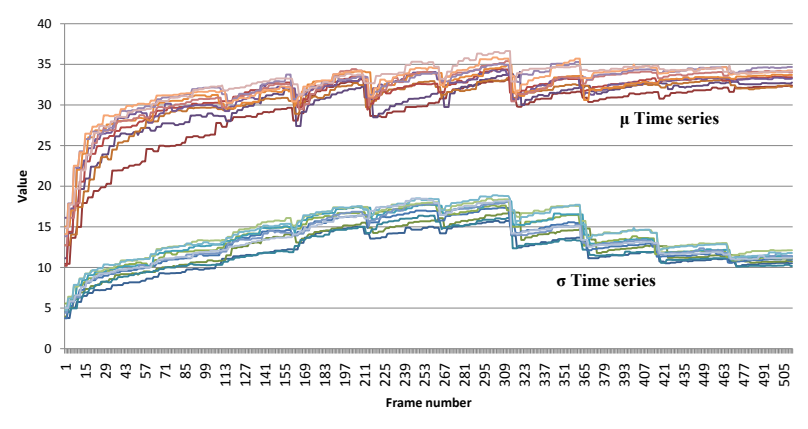

Fig. 7. Different series for $\mu$ (red) and $\sigma$ (blue) describing the palpation procedures of the Full Bottle object. The graphs show that the different time series are consistent despite squeezing the object at different positions.

\section{Classification and distance metric}

We use a $k$-nearest neighbors $(k$-NN) classification method to classify the time series resulting from the palpation procedure. A number of training examples are stored for each object. A new observation is compared to the training data. Based on a distance metric, the $k$-nearest neighbours are found, and the new observation is assigned the label that is most frequent in this set.

In order to calculate the distance between the time series, we use the Dynamic Time Warping algorithm [24], which is widely used in different areas for measuring the similarity between time series by minimizing the effects of distortion and shifts in time or speed. This is important in our case, since we are dealing with real-world perception and action, which both are noisy. The sequences are "warped" nonlinearly in the time dimension to determine a measure of their similarity independent of certain non-linear variations in the time dimension. It allows an elastic transformation and can be used to detect similarity between signals with different phases. Given two time series, $X=\left\{x_{1}, x_{2}, \ldots, x_{N}\right\}, N \in \mathbb{N}$ and $Y=\left\{y_{1}, y_{2}, \ldots, y_{M}\right\}, M \in \mathbb{N}$, the DTW algorithm returns the distance, $d \in \mathbb{R}, d>0$ between the two time series, with $d$ being closer to 0 for more similar time series and larger otherwise.

One palpation procedure, $z$, consists of a $\mu$ and $\sigma$ time series for each of the two fingertip sensors:

$$
z=\left\{Z_{\mu, \text { left }}, Z_{\mu, \text { right }}, Z_{\sigma, \text { left }}, Z_{\sigma, \text { right }}\right\}
$$

where $Z_{\mu, \text { left }}, Z_{\mu, \text { right }}, Z_{\sigma, \text { left }}, Z_{\sigma, \text { right }} \in[0,255]^{N}$ represent the time series of features computed for the images of the left sensor and right sensor.

In the experiments, we consider different classifications methods, based on different distance metrics. The simplest distance metric is based only on the first moment of the sensor on one of the fingertips, e.g., $Z_{\mu, \text { left }}$, to measure the distance between $z_{1}$ and $z_{2}$ :

$$
\operatorname{dist}_{l, 1}\left(z_{1}, z_{2}\right)=D T W\left(Z_{1, \mu, \text { left }}, Z_{2, \mu, \text { left }}\right) \cdot w_{\mu, \text { left }}
$$

where $w_{\mu, \text { left }}$ is used for normalizing the distance metric:

$$
w_{\mu, \text { left }}=1 / \max _{i, j}\left(\operatorname{dist}\left(z_{i}, z_{j}\right)\right),
$$

where $i \neq j, 0<i<n, 0<j<n$.
An improved metric is given by also taking into consideration the second feature:

$$
\begin{array}{r}
\operatorname{dist}_{l, 2}\left(z_{1}, z_{2}\right)=\operatorname{DTW}\left(Z_{1, \mu, \text { left }}, Z_{2, \mu, \text { left }}\right) \cdot w_{\mu, \text { left }}+ \\
D T W\left(Z_{1, \sigma, \text { left }}, Z_{2, \sigma, \text { left }}\right) \cdot w_{\sigma, \text { left }}
\end{array}
$$

The same two distance metrics can be written for the right sensor as well, resulting in $\operatorname{dist}_{r, 1}\left(z_{1}, z_{2}\right)$ and $\operatorname{dist}_{r, 2}\left(z_{1}, z_{2}\right)$.

When considering both sensors, the distance metric can be calculated using only the first feature of both sensors:

$$
\operatorname{dist}_{l r, 1}\left(z_{1}, z_{2}\right)=\operatorname{dist}_{l, 1}\left(z_{1}, z_{2}\right)+\operatorname{dist}_{r, 1}\left(z_{1}, z_{2}\right),
$$

or by taking into consideration both features:

$$
\begin{array}{r}
\operatorname{dist}_{l r, 2}\left(z_{1}, z_{2}\right)=\operatorname{dist}_{l, 1}\left(z_{1}, z_{2}\right)+\operatorname{dist}_{r, 1}\left(z_{1}, z_{2}\right)+ \\
\operatorname{dist}_{l, 2}\left(z_{1}, z_{2}\right)+\operatorname{dist}_{r, 2}\left(z_{1}, z_{2}\right)
\end{array}
$$

These distance metrics are used for our classification algorithm, and we would like to test if the closer to 0 the distance between two observation is, the more likely is the fact that two objects belong to the same observed object.

a)
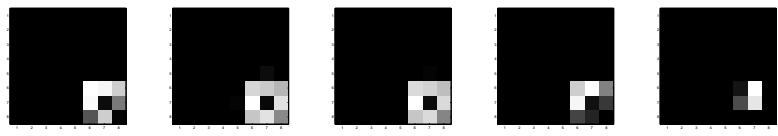

b)
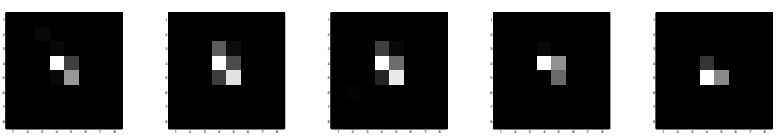

c)
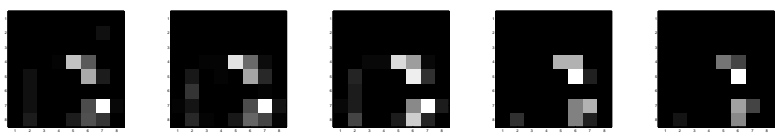

d)
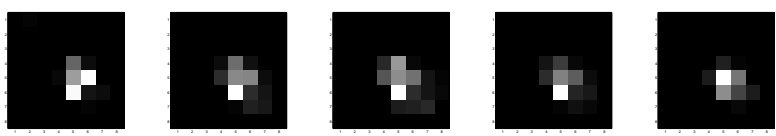

e)
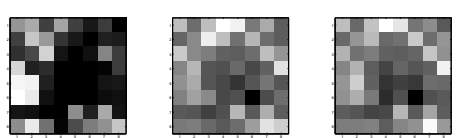

(1s)

(2s)

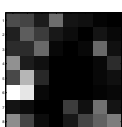

(3s)

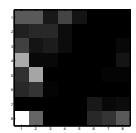

$(4 s)$

Fig. 8. Example tactile images for a few grasped objects, sampled every 1s during the palpation procedure: a)Rubber ball, b)Duck, c)Bad orange, d)Fresh orange and e)Tape

\section{EXPERIMENTAL EVALUATION}

\section{A. Classification Strategy}

For testing our sensor, we have recorded tactile data for 10 various household objects, rigid and deformable, some of them being similar in shape and size to the others (see Figure 6). The set of objects consisted of a rubber ball, a balsam bottle, a rubber duck, an empty 0.51 plastic bottle, a full 0.51 plastic bottle, a bad orange, a fresh orange, a juggling ball, a tape roll and a small block of wood. The tape roll and the block of wood are rigid objects, while the other ones were more or less deformable. Each palpation procedure was repeated around 10 times for each object, and the grasping was executed each time with the object slightly moved or rotated in the gripper jaws, to ensure some 
variability in the data set. We thus obtained a data set of $|\mathcal{D}|=97$ labels for 10 objects. The classification algorithm was based on three $k$-NN classifiers, with $k=1,3,5$. As explained in the previous section, we apply different distance metrics to take into account the tactile readings from the left sensor, from the right sensor and from both sensors. Moreover, the first moment, or the first two moments are used in distance calculation.

In order to quantify the classification performance, we use a 10 -fold cross validation, where we have split $\mathcal{D}$ into 10 disjoint subsets of approximately the same size, $\mathcal{D}_{i}$, each subset having approximately the same number of examples per object, in order to ensure equal training for each label. Each subset is used as a test set, $\mathcal{D}_{\text {test }}=\mathcal{D}_{i}$ where all other 9 subsets are used as training data, $\mathcal{D}_{\text {training }}=\mathcal{D}-\mathcal{D}_{\text {test }}$. The recognition rate of each subset $\mathcal{D}_{i}$ is given by the number of correctly classified labels divided by the total number of tests. We thus obtain 10 recognition rates, one for each fold, $\left\{r_{1}, r_{2}, \ldots, r_{1} 0\right\}$, which we use to calculate the mean and 95\% confidence intervals for the recognition results.

\section{B. Recognition results}

The recognition rates for using one feature (the first moment) for classification are shown in Table I. It can be seen that the recognition rates differ depending on which sensor is used. This is mostly based on the fact that the sensors were placed on the gripper jaws in a non-perfect alignment and because of small differences between the sensors which are due to the manual manufacturing of the sensors. Using both sensors instead of only one improves the performance significantly. This shows that the two sensors compliment each other and make the system more robust. There are no significant differences in recognition performance when a larger $k$ is used in the $k$-NN classifier. This means that we can reduce the computational complexity of the classifier by only considering the first-nearest neighbor without the loss of performance.

Table I also shows the results when two features (first and second moment) are used for classification. Compared to using only one feature, the recognition rates are higher. This and the fact that the difference in performance between the two sensors disappeared shows that the addition of the second feature improves the robustness of the system. Again, no significant differences can be seen for the different values of $k$ in the classifier.

Figure 9 shows the resulting confusion matrices for 1NN classification based on left, right and both sensors, and based on one and two features. The numbers on the axes correspond to the object numbers given in Figure 6. The vertical axis shows the truth and the classification is given on the horizontal axis. In general, it can be observed from the clear diagonal that the classification performs nicely. Some of the objects are harder to recognize, and for example we see that the balsam bottle is classified as either an empty bottle or a bad orange, which shows that they are similar objects. Another case is with the bad orange, that is sometimes classified as a joggling ball due to the fact that both objects are more plastic than elastic, meaning they do not go back to their initial shape that easy. It is possible to see from the tactile images example in Figure 8 that the fresh orange does not become soft as easily and its stiffness determines a more concentrated contact area as well as peak pressure compared to a bad orange. However, bad oranges and fresh oranges are also sometimes hard to distinguish using this method because unless the bad oranges are strongly damaged, they still have a minor elastic behaviour and vice-versa, the fresh oranges have a plastic behaviour after a few palpation procedures.

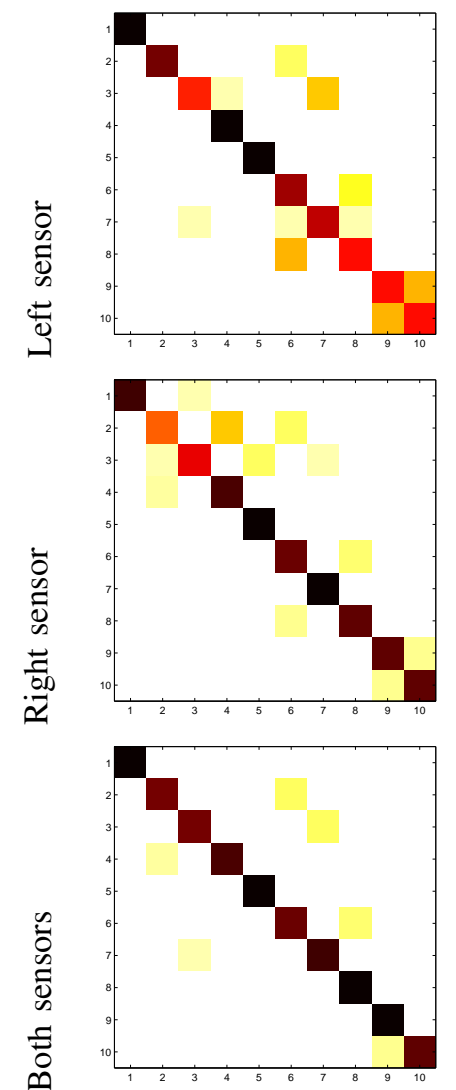

(a)
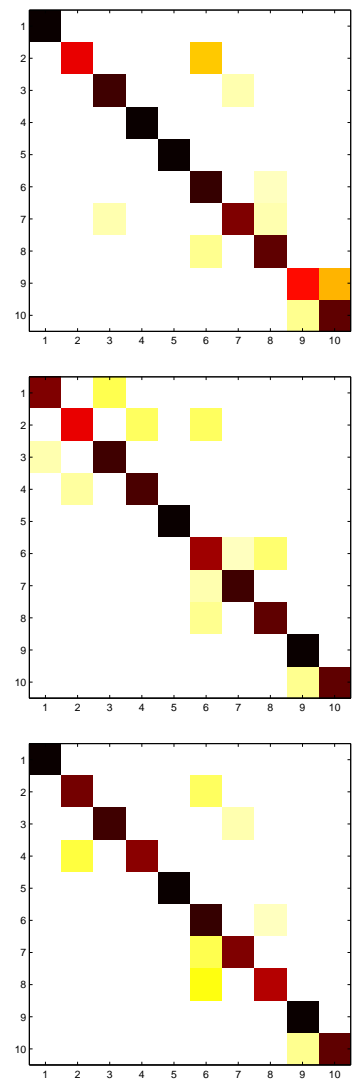

(b)
Fig. 9. Confusion Matrix for 1-NN classification for the proposed sensors: a) using one feature, b) using both features

\section{Comparison with the Weiss Robotics sensor}

We compare our sensor with the Weiss Robotics tactile sensor [10], since this is a widely used sensor for tactile feedback. We therefore investigate if we can obtain similar results using our sensor. The Weiss Robotics tactile sensors are mounted on the Schunk Dextrous Hand SDH and they consist of 13 rows x 6 columns of taxels, in about $24 \mathrm{~mm}$ x $51 \mathrm{~mm}$ area (see Figure 10). The area occupied by this sensor is almost double compared to the sensor that we propose, therefore a different size resolution is achieved. Increasing the geometrical resolution of our sensor would mean the addition of detail data for the extra cells. Such details could be used for improvement of recognition if the objects would be small enough to actually benefit from a more precise contact shape. Given the fact that the objects are 
TABLE I

CLASSIFICATION RESULTS FOR ONE AND TWO FEATURES USING 10 FOLD CROSS VALIDATION, 95 \% CONFIDENCE INTERVAL

Using one feature

\begin{tabular}{|c|c|c|c|}
\hline$k N N$ & Left Sensor & Right Sensor & Both Sensors \\
\hline 1NN & $78.14 \pm 4.55 \%$ & $86.00 \pm 6.90 \%$ & $91.57 \pm 3.89 \%$ \\
3NN & $76.14 \pm 6.86 \%$ & $86.00 \pm 6.32 \%$ & $88.00 \pm 4.64 \%$ \\
$5 N N$ & $70.71 \pm 5.40 \%$ & $84.00 \pm 6.90 \%$ & $84.00 \pm 6.32 \%$ \\
\hline
\end{tabular}

Using two features

\begin{tabular}{|c|c|c|c|}
\hline$k N N$ & Left Sensor & Right Sensor & Both Sensors \\
\hline 1NN & $88.57 \pm 5.18 \%$ & $88.00 \pm 6.07 \%$ & $92.00 \pm 4.64 \%$ \\
3NN & $87.00 \pm 6.23 \%$ & $85.00 \pm 6.93 \%$ & $90.00 \pm 5.54 \%$ \\
5NN & $78.14 \pm 7.69 \%$ & $85.00 \pm 6.93 \%$ & $91.00 \pm 5.15 \%$ \\
\hline
\end{tabular}

rather big with respective to the size of one cell and because of the dimensionality reduction, this resolution difference would not influence the comparison in classification results between the two sensors. However, other applications could benefit from more detailed data regarding contact shapes. We tested this sensor using the same experimental setup. Again we test the recognition performance on the 10 objects, each object with 10 observations. After processing the recorded tactile data according to the same algorithms described for our sensor, we obtained a similar data set of time series of features.

The results shown in Table II suggest that our sensor performs similar to the Weiss sensor. Using our classification procedure it is also possible to discriminate with high recognition rates between the chosen objects using the Weiss sensor. Looking at the differences between left and right sensor, the experiments show that the Weiss sensors are also prone to manufacturing and placement differences. This is caused by the sensitivity of the specific sensor used, which was rather low, caused probably by manufacturing or wear and tear of the sensor during it's use with the robotic hand.

The confusion matrices shown in Figure 11 show that the recognition results are in general good, pointed out by the clear diagonal. However, some objects were not as easy to recognize as others and rigid objects, such as the tape or the wood block which were classified sometimes as the rubber ball or the balsam bottle. This is understandable because they are almost rigid and the palpation procedure would sometimes start only after establishing a good contact with the object. The sensitivity of the sensors was the limiting factor because it delayed the start of the palpation procedure to the point where the object was already deformed. On the other hand, even though using our sensor we have similar recognition rates, we have obtained rather different results in the confusion matrix, where we could see that deformable objects such as good oranges, bad oranges and joggling balls were confused more often. Rigid objects were also prone to confusion, but were only confused with other rigid objects. This different behaviour is based on the increased sensitivity in our sensor and it suggests that for better classification rates we should take into consideration other features extracted
TABLE II

CLASSIFICATION RESULTS USING WEISS ROBOTICS SENSORS, FOR ONE AND TWO FEATURES USING 10 FOLD CROSS VALIDATION, $95 \%$ CONFIDENCE INTERVAL

Using one feature

\begin{tabular}{|c|c|c|c|}
\hline$k N N$ & Left Sensor & Right Sensor & Both Sensors \\
\hline 1NN & $87.00 \pm 6.23 \%$ & $75.00 \pm 6.35 \%$ & $91.00 \pm 6.47 \%$ \\
3NN & $85.00 \pm 6.35 \%$ & $69.00 \pm 3.34 \%$ & $88.00 \pm 8.22 \%$ \\
$5 \mathrm{NN}$ & $81.00 \pm 7.04 \%$ & $67.00 \pm 4.84 \%$ & $84.00 \pm 7.94 \%$ \\
\hline
\end{tabular}

Using two features

\begin{tabular}{|c|c|c|c|}
\hline$k \mathrm{NN}$ & Left Sensor & Right Sensor & Both Sensors \\
\hline 1NN & $93.00 \pm 4.84 \%$ & $74.00 \pm 7.44 \%$ & $92.00 \pm 4.64 \%$ \\
3NN & $88.00 \pm 7.23 \%$ & $72.00 \pm 7.74 \%$ & $85.00 \pm 7.96 \%$ \\
$5 \mathrm{NN}$ & $84.00 \pm 6.32 \%$ & $69.00 \pm 5.15 \%$ & $84.00 \pm 7.44 \%$ \\
\hline
\end{tabular}

from the tactile images.

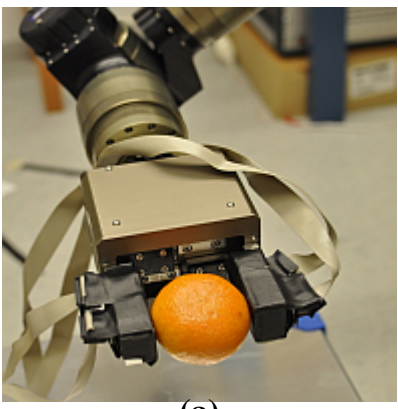

(a)

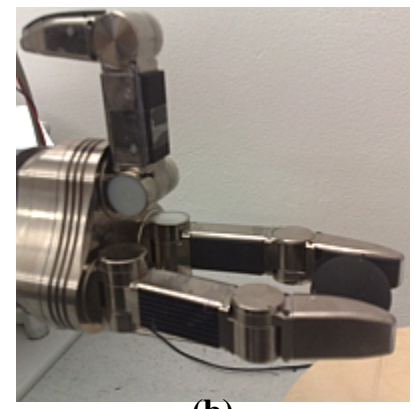

(b)
Fig. 10. Experimental setup a) using the Schunk Parallel Gripper with our sensor, b) using the Schunk Dextrous Hand with Weiss sensors

\section{CONCLUSIONS AND FUTURE WORK}

In this paper, we have described the building principles of a novel flexible array tactile sensor and its use for recognition of different household objects based on their haptic feedback when grasped. Starting from describing the working principles of the piezoresistive materials we continue by showing how to build a tactile-array sensor that is flexible, very sensitive and cheap to manufacture and we also describe the implementation of a data acquisition system for the proposed sensor. We furthermore tested the usefulness of the sensor by classifying different grasped soft and rigid objects based only on their tactile feedback. We use dynamic time warping to compare the similarity in the signal based on basic features extracted from a series of tactile images with a $k$-nearest neighbor classifier. We used time series of two features for the classification: the first moment and the second moment of the tactile images. We showed how the classification rates can be improved by combining the two features. By comparing the results obtained with the proposed tactile sensor with the results obtained with a Weiss Robotics tactile sensor, we conclude that the proposed sensor performs at least as good, while having other advantages such as being cheap, more sensitive and flexible. Even though the tests for the considered application were done using flat 


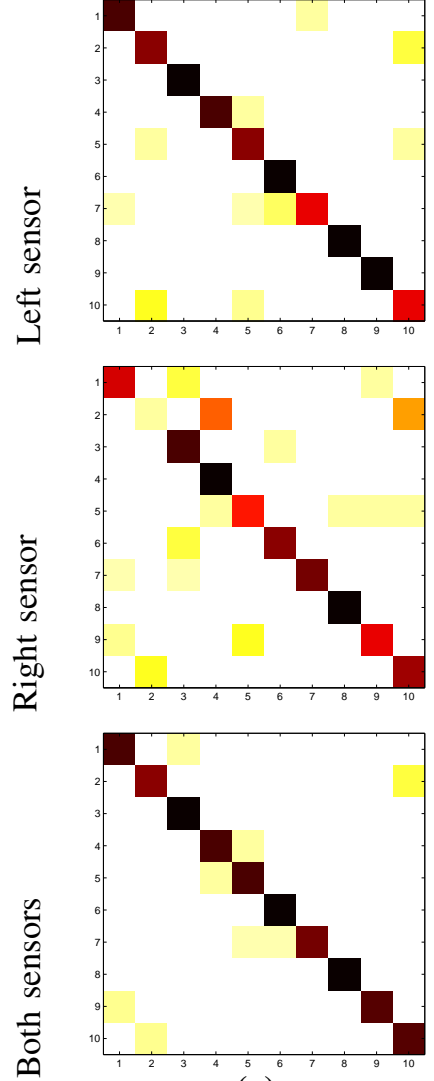

(a)
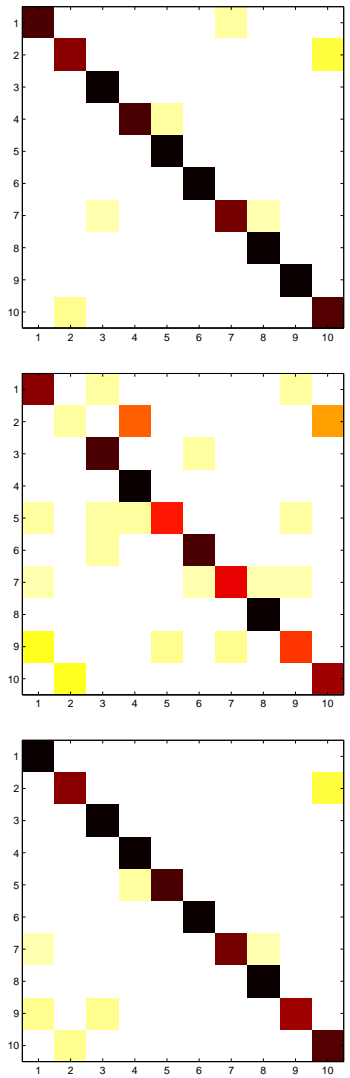

(b)
Fig. 11. Confusion Matrix for 1-NN classification for Weiss Robotics sensors: a) using one feature, b) using both features

jaws, the flexibility of the sensor ensures applicability over curved surfaces. We are confident that our sensor prototype can be successfully used in robotic applications such as grasping unknown objects and determining some physical characteristics of these objects, such stiffness and texture.

Although we demonstrated good results in terms of recognition rates, future work will consider an improved version of the sensor, with increased resolution that uses a different manufacturing method, ensuring a good repeatability as well as similar response from all the taxels. A larger data set with a greater variation in number of the samples will also be considered Other applications are also envisioned, such as recognizing objects based on their contact geometrical features.

\section{ACKNOWLEDGMENTS}

This work is supported by the Danish Handyman Project, a Danish initiative with partners from both academia and industry that tries to address advanced robot grasping in industrial environments, by the EU through the project eSMCs, IST-FP7-IP-270212, and by the Swedish Foundation for Strategic Research.

\section{REFERENCES}

[1] A. Saxena, J. Driemeyer, and A. Y. Ng, "Robotic grasping of novel objects using vision," International Journal of Robotics Research, vol. 27 , no. 2, pp. 157-173, 2008.
[2] R. Detry, E. Baseski, M. Popovic, Y. Touati, N. Krueger, O. Kroemer, J. Peters, and J. Piater, "Learning continuous grasp affordances by sensorimotor exploration," in From Motor Learning To Interaction Learning in Robots, O. Sigaud and J. Peters, Eds. Berlin, Germany: Springer-Verlag, 2010.

[3] K. Huebner, K. Welke, M. Przybylski, N. Vahrenkamp, T. Asfour, D. Kragic, and R. Dillmann, "Grasping known objects with humanoid robots: A box-based approach," in 14th International Conference on Advanced Robotics, Munich, Germany, June 2009.

[4] B. Rasolzadeh, M. Bjorkman, K. Huebner, and D. Kragic, "An active vision system for detecting, fixating and manipulating objects in real world," International Journal of Robotics Research, vol. 29, no. 2-3, pp. 133-154, 2010.

[5] M. Popovic, D. Kraft, L. Bodenhagen, E. Baseski, N. Pugeault, D. Kragic, T. Asfour, and N. Kruger, "A strategy for grasping unknown objects based on co-planarity and colour information," Robotics and Autonomous Systems, vol. 58, no. 5, pp. 551-565, 2010.

[6] J. Bohg and D. Kragic, "Learning grasping points with shape context," Robotics and Autonomous Systems, vol. 59, no. 4, pp. 362-377, 2010.

[7] M. Shimojo, T. Araki, A. Ming, and M. Ishikawa, "A high-speed mesh of tactile sensors fitting arbitrary surfaces," IEEE SENSORS JOURNAL, vol. 10, no. 4, pp. 822-830, 2010.

[8] M. Higashimori, M. Kaneko, A. Namiki, and M. Ishikawa, "Design of the $100 \mathrm{~g}$ capturing robot based on dynamic preshaping," International Journal of Robotics Research, vol. 24, no. 9, pp. 743-753, 2005.

[9] Y. Bekiroglu, D. Kragic, and V. Kyrki, "Learning grasp stability based on tactile data and hmms," in Proceedings of the 19th IEEE International Symposium in Robot and Human Interactive Communication, 2010.

[10] "Weiss robotics tactile sensor," [Online], http://www.weiss-robotics. de/en.html.

[11] M.H.Lee and H. Nicholls, "Tactile sensing for mechatronics - a state of the art survey," Mechatronics, vol. 9, pp. 1-32, 1999.

[12] M. Cutkosky, R. Howe, and W. Provancher, "Force and tactile sensors," Springer Handbook of Robotics, pp. 455-476, 2008.

[13] K. Weiss and H. Woern, "The working principle of resistive tactile sensors cells," Proc. IEEE International Conference on Mechatronics and Automation, 2005.

[14] P. Goethals, "Tactile feedback for robot assisted minimally invasive surgery:an overview," Department of Mechanical Engineering K.U.Leuven, Tech. Rep., 2008.

[15] D. Goeger, N. Gorges, and H. Woern, "Tactile sensing for an anthropomorphic robotic hand: Hardware and signal processing," in IEEE International Conference on Robotics and Automation, 2009.

[16] M. Shimojo, A. Namiki, M. Ishikawa, R. Makino, and K. Mabuchi, "A tactile sensor sheet using pressure conductive rubber with electricalwires stitched method," IEEE SENSORS JOURNAL, vol. 4, no. 5, October 2004.

[17] S. Chitta, M. Piccoli, and J. Sturm, "Tactile object class and internal state recognition for mobile manipulation," in International Conference on Robotics and Automation, 2010.

[18] A. Schneider, J. Sturm, C. Stachniss, M. Reisert, H. Burkhardt, and W. Burgard, "Object identification with tactile sensors using bag-offeatures," in IEEE/RSJ International Conference on Intelligent Robots and Systems, 2009.

[19] A. Morales, M. Prats, P. Sanz, and A. P. Pobil, "An experiment in the use of manipulation primitives and tactile perception for reactive grasping," in Science and Systems, Workshop on Robot Manipulation: Sensing and Adapting to the Real World, Atlanta, USA, 2007.

[20] M. Prats, P. Sanz, and A. del Pobil, "Vision-tactile-force integration and robot physical interaction," in IEEE International Conference on Robotics and Automation, Kobe, Japan, 2009, pp. 3975-3980.

[21] A. Bierbaum, M. Rambow, T. Asfour, and R. Dillmann, "A potential field approach to dexterous tactile exploration," in IEEE/RAS International Conference on Humanoid Robots (Humanoids), 2008.

[22] A. M. Howard and G. A. Bekey, "Intelligent learning for deformable object manipulation," Autonomous Robots, vol. 9, no. 1, pp. 51-58, 2000.

[23] A. Drimus, N. Marian, and A. Bilberg, "Tactile sensing for object identification," in Research and Education in Mechatronics, Glasgow, UK, 2009.

[24] H. Sakoe and S. Chiba, "Dynamic programming algorithm optimization for spoken word recognition," Acoustics, Speech and Signal Processing, IEEE Transactions on, vol. 26, no. 1, pp. 43-49, 1978. 